\title{
The Rotavirus Surveillance System in Yemen: Evaluation Study
}

Eman Abdullah Lardi ${ }^{1}$, MBBCH; Sharaf Sharaf Al Kuhlani ${ }^{2}$, BSc; Mohammed Abdullah Al Amad ${ }^{1}$, MPH; Abdulwahed Abduljabar Al Serouri ${ }^{1}, \mathrm{PhD}$; Yousef Saleh $\mathrm{Khader}^{3}, \mathrm{ScD}$

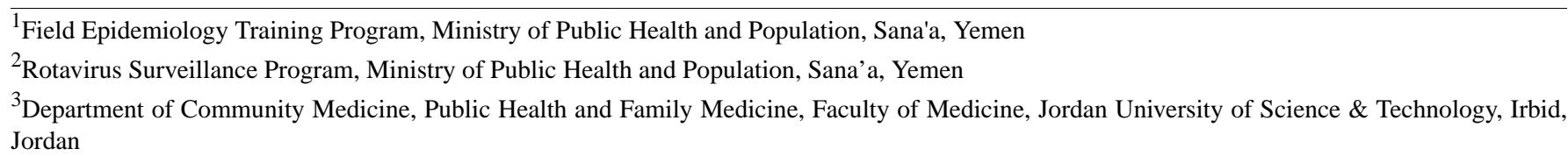

Corresponding Author:

Eman Abdullah Lardi, MBBCH

Field Epidemiology Training Program

Ministry of Public Health and Population

AL-Hasaba, Mazda street

Sana'a

Yemen

Phone: 967771866574

Email: eman2020-2020@ hotmail.com

\section{Abstract}

Background: Rotavirus (RV) kills over 185,000 children <5 years every year and is responsible for over one-third of all child diarrheal deaths worldwide. The Rotavirus Surveillance System (RVSS) in Yemen was launched in 2007 at five sentinel sites to monitor the impact of the vaccine on RV morbidity and mortality.

Objective: This study aimed to determine the usefulness of the RVSS, assess its performance, and identify the strengths and weaknesses of its implementation.

Methods: The Centers for Disease Control and Prevention's updated guidelines on evaluating a public health surveillance system were used to evaluate the RVSS. In this assessment, qualitative indicators, such as usefulness, flexibility, stability, simplicity, and acceptability, were assessed through in-depth interviews with stakeholders at the central level and semistructured questionnaires with the sentinel site coordinators. The indicators for quantitative attributes-sensitivity, positive predictive value (PPV), completeness, and timeliness - were assessed by reviewing the results of laboratory samples and a random sample of case report forms. The scores for the indicators were expressed as poor $(<60 \%)$, average $(60 \%$ to $<80 \%)$, and good $(\geq 80 \%)$.

Results: The overall usefulness score of the RVSS was $73 \%$, indicating an average rank. The RVSS was rated as having good flexibility (91\%) and stability (81\%), and average simplicity (77\%) and acceptability (76\%). In terms of quantitative attributes, the system was poor for sensitivity (16\%), average for PPV (73\%), and good for completeness (100\%) and timeliness (100\%).

Conclusions: Although the system attributes were flexible, stable, capable of providing quality data, and performing timely data reporting, some attributes still needed improvements (eg, usefulness, simplicity, acceptability, and PPV). There is a need for a gradual replacement of donor funds with government funds to ensure sustainability. The RVSS in Yemen strongly requires a progressive increase in the number of sites in governorates and sensitivity enhancement.

(JMIR Public Health Surveill 2021;7(6):e27625) doi: 10.2196/27625

\section{KEYWORDS}

Rotavirus; surveillance system; evaluation, Yemen

\section{Introduction}

Rotavirus (RV) is the major cause of vaccine-preventable severe and fatal diarrhea among young children [1]. Infection can be asymptomatic, cause mild to moderate gastroenteritis, or severe gastroenteritis with dehydration requiring hospitalization [2].
Recovery from a first RV infection usually does not lead to permanent immunity, and reinfection can occur at any age but with less severity than the first. The World Health Organization (WHO) identified an RV vaccine as the key strategy in reducing the RV-diarrhea burden. The monovalent (RV1) Rotarix and the pentavalent (RV5) Rota Teg are two safe and effective oral vaccines against $\mathrm{RV}$ infection in children [3]. 
Globally, nearly every child in the world gets infected with RV between 3 and 5 years of age. However, the highest rates of severe disease occur commonly at the age of 6 to 24 months $[3,4]$. Studies in the Eastern Mediterranean Region (EMR) have estimated approximately 65,000 child deaths each year due to RV infection. Mortality remains high in this region, especially in countries with a lower per capita income, such as Pakistan, Afghanistan, Sudan, Yemen, and Somalia [5]. The countries with a higher per capita income have few deaths, but the burden of severe RV disease is reflected in the many hospitalizations and clinic visits among children $<5$ years of age [5].

Yemen is a resource-limited country with acute gastroenteritis-related morbidity and mortality as the major health problem. The Ministry of Public Health and Population (MoPH\&P) had introduced the RV vaccine into the routine immunization schedule in 2012. The vaccine is administered in two doses: the first dose is administrated at 6 weeks of age, and the second dose is completed by 10 weeks of age [6]. The introduction of the RV vaccine helped to decrease the burden of severe RV gastroenteritis and RV-associated mortality [7]. The RV hospitalization incidences in Yemen decreased from $43.8 \%$ in 2009 to $10.5 \%$ in 2014 [7].

The Rotavirus Surveillance System (RVSS) was launched in 2007 at five sentinel sites to monitor the impact of the vaccine on RV morbidity and mortality. The RVSS has never been evaluated before in Yemen. Therefore, this study aimed to determine the usefulness and performance of the RVSS, and identify the strengths and weaknesses of the system implementation.

\section{Methods}

\section{Evaluation Design}

The Centers for Disease Control and Prevention's (CDC) updated guidelines on evaluating a public health surveillance system were used to evaluate the RVSS [8]. In this assessment, qualitative indicators of usefulness and other attributes of the system (eg, flexibility, stability, simplicity, and acceptability) were assessed through in-depth interviews with stakeholders at the central level, and semistructured questionnaires were used with the sentinel site coordinators. Furthermore, the indicators for quantitative attributes such as sensitivity, positive predictive value (PPV), completeness, and timeliness were assessed by reviewing the results of laboratory samples and a random sample review of case report forms. All the five sentinel sites covered by the RVSS (Yemen Swedish Hospital in Taiz, Al Wahda General Teaching Hospital in Aden, Al Sabeen Maternal Hospital in Sana'a, Al-Thawra Hospital in Ibb, and Al-Thawra Hospital in Al Hudaydah) were included and established for qualitative evaluation. The RVSS evaluation was conducted from October to December 2018.

\section{Evaluation Approach}

The RVSS stakeholders at the central level and the sentinel site coordinators were included in this study. Different data collection methods were used, such as a desk review of the RVSS documents, in-depth interviews with stakeholders at the central level, and semistructured questionnaires with the sentinel site coordinators. The evaluation involved reviewing the available documents such as operational manuals, monthly and annual reports, and databases. The documents were reviewed before interviewing the stakeholders to obtain information about the RVSS. Seven in-depth interviews were conducted with the stakeholders at the central levels to understand the RVSS implementation, as well as its usefulness, flexibility, stability, and strengths and weaknesses. The indicators for usefulness and other qualitative attributes were developed according to the CDC guidelines. A registers review was used to assess the quantitative attributes (sensitivity, PPV, completeness, and timeliness).

The indicators of attributes (usefulness, flexibility, and stability of the system) were assessed using questions with "yes" or "no" answers that were scored as 1 or 0 , respectively. The level of simplicity and acceptability of the system was assessed on a 5-point Likert scale ( 1 =strongly disagree, $2=$ disagree, $3=$ neutral, 4=agree, and 5=strongly agree).

For each indicator, the score percent was calculated as:

$\underline{\text { Sum of all respondents' scores for each indicator }}$ $\times 100$ Maximum score for indicator $\times$ Number of respondents

The overall attribute score percent was calculated as:

Sum of all respondents' scores for all indicators Number of indicators $\times$ Maximum score for indicator $\times$ Number of respondents $\times 100$

The sensitivity of the system was assessed by the proportion of stool samples of the suspected cases of RV gastroenteritis that tested positive for RV. The PPV was calculated as the proportion of the positive RV stool samples reported by the sentinel sites that tested positive at the National Central Public Health Laboratory (NCPHL). Timeliness was measured as the proportion of reports sent to the central level by the deadline. Missing data were measured by selecting the 1-year data and calculating the percentages of the missed variables. The data accuracy was assessed by comparing reports at the central level with the case report form. The ranking and scoring system used for the quantitative and qualitative attributes, as well as for the indicators of each attribute, were as follows: poor $(<60 \%)$ average $(60 \%$ to $<80 \%)$, and good $(\geq 80 \%)$.

\section{Ethical Approval}

The ethical review committee of MoPH\&P advised that ethical approval for this evaluation protocol was not needed as it was part of the ongoing national evaluation activity. The stakeholders at the central level and the sentinel site coordinators were explained the aim of the study and were requested to participate. If they agreed, either an interview was conducted or a semistructured questionnaire was administered. Confidentiality of the collected data was maintained by limiting access to the research team only.

\section{Results}

\section{Description of the RVSS}

The MoPH\&P established the RVSS in 2007 with technical and financial support from the WHO. After the RV vaccines were introduced in Yemen in 2012, the objectives of the RVSS were 
updated to include assessments of the vaccine impact on RV morbidity and mortality among children $<5$ years, as well as changes in RV epidemiology and circulating strains, and provide a basis for further epidemiologic research.

The RVSS required collecting data on individual cases of diarrhea among children $<5$ years of age. It was the active surveillance at the five sentinel sites. The potential data sources included inpatients in the Department of Pediatrics, the Emergency Department, and the Diarrhea Treatment Centers.
A suspected case was a case with an acute ( $<14$ days) watery diarrhea, defined as 3 or more loose or watery stools within a 24-hour period in a child $<5$ years of age admitted for diarrhea treatment into the hospital ward or the emergency unit at the sentinel sites. Children with bloody diarrhea and nosocomial infections were excluded. A confirmed case was a suspected case with the presence of RV in its stool confirmed by an enzyme immunoassay (EIA) or polymerase chain reaction (PCR)-based methods. Figure 1 shows the RVSS data collection flow chart.

Figure 1. Flow chart for RVSS data collection methods employed in phases 1 and 2. CDC: Centers for Disease Control and Prevention, EMRO: Regional Office for the Eastern Mediterranean, MoPH\&P: Ministry of Public Health and Population, NCPHL: National Central Public Health Laboratory, RVSS: Rotavirus Surveillance System, WHO: World Health Organization.

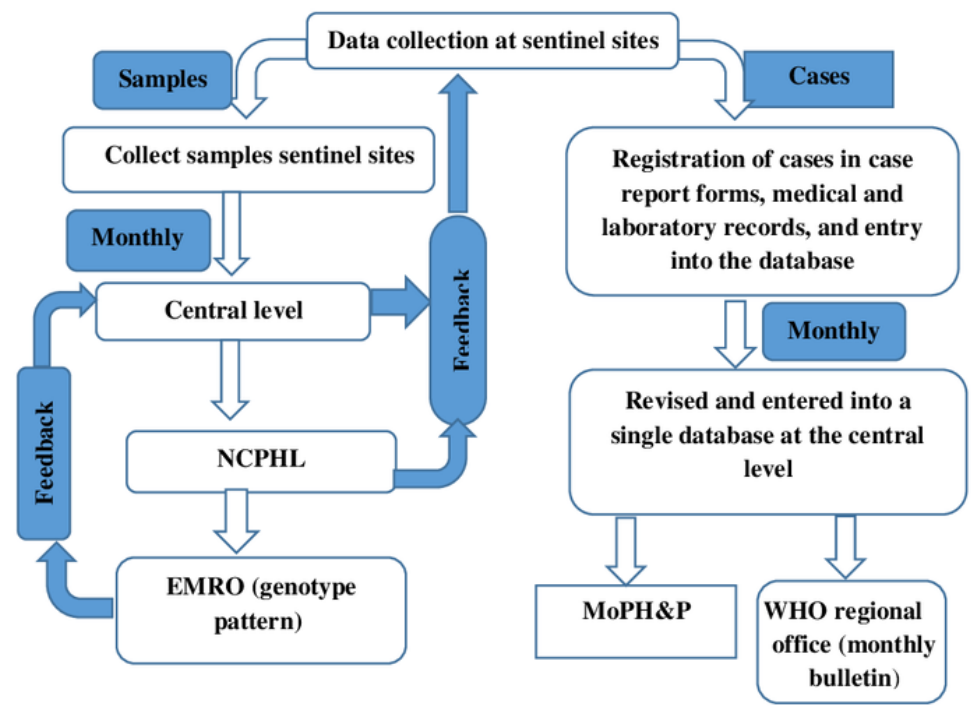

In-depth Interviews With Stakeholders at the Central Level

\section{Usefulness}

Table 1 highlights that 5 out of 8 usefulness indicators achieved a good rank, while 2 indicators, development of the national policy strategy for the national immunization program and estimation of the RV magnitude, incidence, and mortality, achieved a poor rank. Another indicator, planning the resources, achieved an average rank. The overall usefulness indicated an average $\operatorname{rank}(\mathrm{n}=41,73 \%)$.

Table 1. The scores (score percent, \%) and rank of the usefulness indicators as assessed by the central-level stakeholders.

\begin{tabular}{lll}
\hline Indicator & Score $(\%)$ & Rank \\
\hline $\begin{array}{l}\text { The system data provide an estimate of rotavirus magnitude, incidence, } \\
\text { and mortality }\end{array}$ & $2(29)$ & Poor \\
$\begin{array}{l}\text { The system data detect trends of rotavirus spread over time } \\
\text { The system data recognize high-risk groups }\end{array}$ & $7(100)$ & Good \\
The system data plan the resources for prevention and control & $5(71)$ & Good \\
$\begin{array}{l}\text { The system data update and develop the national policy strategy for the } \\
\text { national immunization program }\end{array}$ & $2(29)$ & Poor \\
The system data assess the effect of interventions & $6(86)$ & Good \\
The system data estimate the needs of laboratory kits & $6(86)$ & Good \\
The system data are used as the basis for epidemiologic research & $6(86)$ & Good \\
Overall usefulness & $41(73)$ & Average
\end{tabular}




\section{Flexibility}

Table 2 shows that 5 out of 6 flexibility indicators achieved a good rank, while the indicator, "The system can accommodate any changes in funding," was ranked poor. The overall flexibility achieved a good rank $(\mathrm{n}=38,91 \%)$.

Table 2. The scores (score percent, \%) and rank of the flexibility indicators as assessed by the central-level stakeholders.

\begin{tabular}{lll}
\hline Indicator & Score $(\%)$ & Rank \\
\hline The system can accommodate changes in the number of sentinel sites & $7(100)$ & Good \\
$\begin{array}{l}\text { The system can accommodate any changes in funding } \\
\text { The system can accommodate changes in case definition }\end{array}$ & $4(57)$ & Poor \\
$\begin{array}{l}\text { The system can accommodate changes in reporting method } \\
\text { The system can be adapted to integrate with other surveillance systems }\end{array}$ & $7(100)$ & Good \\
$\begin{array}{l}\text { The system can accommodate changes in data with minimum cost and } \\
\text { efforts }\end{array}$ & $7(100)$ & Good \\
Overall flexibility & $38(91)$ & Good \\
\hline
\end{tabular}

\section{Stability}

The study showed that 4 out of the 6 stability indicators achieved a good rank (Table 3). However, 2 indicators, the availability of planned resources for maintenance and the sustainability of the system if donors withdrew their support, achieved a poor rank. The overall stability ranked good $(n=34,81 \%)$.

Table 3. The scores (score percent, \%) and rank of the stability indicators as assessed by the central-level stakeholders.

\begin{tabular}{lll}
\hline Indicator & Score $(\%)$ & Rank \\
\hline No unscheduled system outages occurred during the last month & $7(100)$ & Good \\
No electrical power outage occurred during the last week & $6(86)$ & Good \\
There are planned resources for the maintenance of the system & $4(57)$ & Poor \\
The system is stable even after the sponsor's withdrawal of support & $3(43)$ & Poor \\
The system does not require time to manage data & $7(100)$ & Good \\
Reports are released regularly & $34(100)$ & Good \\
Overall stability & $34)$ & Good \\
\hline
\end{tabular}

\section{Strengths and Weaknesses}

The majority of stakeholders $(n=6,86 \%)$ reported the presence of a qualified medical team at the central and terminal levels as one of the strengths of the RVSS. Around half $(n=4,57 \%)$ respondents said the accuracy of data as an important strength:

Another strength was the continuity of data flow to the central level.

Regarding the weaknesses in the RVSS, all stakeholders said that a lack of financial support from the government and total dependence on the WHO for support were the major weaknesses of the system. The small number of surveillance sites was another reported weakness. One participant said:

Other weaknesses included delays in sending the feedback about the samples' results by the WHO Regional Office for the Eastern
Mediterranean (WHO EMRO), lack of laboratory kits, and poor utilization of research findings.

\section{Semistructured Questionnaire With the Sentinel Site Coordinators}

The five sentinel site coordinators (all were females) responded to the semistructured questionnaire.

\section{Simplicity}

Table 4 shows that 8 out of 10 simplicity indicators (eg, case definition is available and easy to use, less time spent on collecting data, etc) achieved a good rank, while 2 indicators, the availability of laboratory tests and training, achieved a poor rank, and the overall simplicity was ranked average $(n=193$, $77 \%)$. 
Table 4. The scores (score percent, \%) and rank of the simplicity indicators as assessed by the sentinel site coordinators.

\begin{tabular}{lll}
\hline Indicator & Score $(\%)$ & Rank \\
\hline The rotavirus case definition is available & $25(100)$ & Good \\
The rotavirus case definition is easy to use & $24(96)$ & Good \\
The case report form is available & $24(96)$ & Good \\
The case report form is easy to use & $24(96)$ & Good \\
Less time spent on collecting data & $20(80)$ & Good \\
Transmitting data to the enteral level is easy & $22(88)$ & Good \\
Follow-up of cases is easy & $20(80)$ & Good \\
Laboratory tests available in the health facility to confirm a diagnosis & $5(20)$ & Poor \\
You received training for rotavirus surveillance & $22(88)$ & Good \\
Training courses are conducted frequently & $7(28)$ & Poor \\
Overall simplicity & $193(77)$ & Average \\
\hline
\end{tabular}

\section{Acceptability}

Table 5 displays the 4 acceptability indicators used in the study. It was found that the indicators willingness to participate in the RVSS and responsiveness of the system to suggestions achieved

a good rank. However, satisfaction with the RVSS and receiving feedback from the central level achieved average and poor ranks, respectively. The overall acceptability achieved an average rank $(\mathrm{n}=76,76 \%)$.

Table 5. The scores (score percent, \%) and rank of the acceptability indicators as assessed by the sentinel site coordinators.

\begin{tabular}{lll}
\hline Indicator & Score (\%) & Rank \\
\hline You are willing to participate in the Rotavirus Surveillance System & $24(96)$ & Good \\
You are satisfied with the surveillance system & $17(68)$ & Average \\
Received feedback report from the central level & $12(48)$ & Poor \\
Responsiveness of the system to suggestions & $23(92)$ & Good \\
Overall acceptability & $76(76)$ & Average \\
\hline
\end{tabular}

\section{Assessment of Quantitative Attributes: Sensitivity, PPV, Completeness, and Timeliness}

Of the 1787 cases suspected of having RV gastroenteritis at the sentinel sites, 1542 had their stool samples tested. Of the total cases tested for the stool samples, only $16 \%(\mathrm{n}=244)$ samples tested positive for RV, indicating poor sensitivity. However, for PPV, about $73 \%(n=178)$ of the positive RV stool samples reported by the sentinel site tested positive at the NCPHL.
There were no missing variables when a random sample of 30 case report forms was reviewed. These forms were found to be consistent with the database. Therefore, completeness was $100 \%$ $(n=30)$. All sentinel sites sent their reports by the fifth day of next month. Therefore, timeliness was also $100 \%(n=5)$.

\section{Overall Performance of the RVSS}

The overall RVSS performance was found to be average (Table $6)$.

Table 6. Summary of the overall performance of the Rotavirus Surveillance System (RVSS).

\begin{tabular}{lll}
\hline Attributes & Score $(\%)$ & Rank \\
\hline Performance according to the central level & $113(81)$ & Good \\
Performance according to sentinel sites & $269(77)$ & Average \\
Performance of quantitative attributes & $215(57)$ & Poor \\
Overall RVSS performance & $597(69)$ & Average \\
\hline
\end{tabular}

\section{Discussion}

\section{Principal Findings}

The RVSS performance evaluation could enhance the usefulness of the surveillance data for public health action. In this evaluation, we assessed the attributes and operation of the RVSS in Yemen using the CDC's updated guidelines [8]. The RVSS data helped estimate the RV severity and provided a basis for epidemiologic research. However, it was reported that the RVSS data were used poorly to update and develop the national policy strategy for the national immunization program in Yemen. In contrast, the Australian Rotavirus Serotyping Program evaluation showed good usefulness of the system [9]. 
The flexibility of the RVSS was rated as good, and the system appeared to be able to adapt and accommodate new changes such as changes in the RV reporting method (phone reporting) and changes in the case definition. However, the system was found to be considerably donor dependent and could poorly accommodate any changes in funding. These observations were different from the evaluation results of the Australian Rotavirus Serotyping Program, which showed the system to be flexible and able to adapt to the changes [9]. Similarly, the RVSS evaluation in Kenya demonstrated the system to be flexible as it could incorporate new reporting sources [10].

The stability of the RVSS was rated good in this study. Although the system was stable and did not require time to manage the data, the system was considered poorly stable if the donors withdrew their support. The RVSS simplicity was rated average, while the case definition and the surveillance case report forms were reported to be available and easy to use. However, the laboratory tests in the health facility were not available to confirm a diagnosis. A comparison with the systems available in other countries showed that the Kenya RVSS scored better on simplicity [10].

The acceptability of the RVSS was rated as average, reflected by the stakeholders' willingness to participate in the RVSS and the responsiveness of the system to suggestions. However, the stakeholders' satisfaction with the RVSS and receiving feedback from the central level achieved average and poor rankings, respectively. For the last 2 years, the system did not receive any feedback reports from the WHO EMRO laboratories.

The sensitivity of the system was poor as only $16 \%$ of stool samples tested positive, which was contrary to a previous evaluation from Australia that found the system sensitive [9].
Moreover, the PPV in this evaluation was $73 \%$, whereas a PPV of $98.5 \%$ was reported from the Kenyan RVSS evaluation [10]. Completeness was $100 \%$, which was slightly higher than the results reported in the Kenya RVSS evaluation, where the completeness was $88 \%$ [10].

Our evaluation had several limitations. The assessment did not include one of the essential attributes of the system, representativeness, because the system depended on only five sentinel sites in five governorates and did not include the rest of the governorates or health facilities. Furthermore, we could not assess the timeliness regarding the feedback on the samples from the WHO EMRO during the period 2017-2018 because the WHO stopped receiving samples since 2017. Regarding the sensitivity and PPV, our evaluation was based only on the results of the 2017-tested samples. The sensitivity of the 2018 samples was not evaluated because the samples were not tested due to a lack of laboratory kits.

\section{Conclusions}

Although the system attributes were flexible, stable, capable of providing quality data, and performing timely data reporting, some attributes still needed improvement (eg, usefulness, simplicity, acceptability, and PPV). The system sustainability requires planning a gradual replacement of donor funds with government funds. Additionally, it is imperative that the NCPHL be upgraded with a RV genotype testing facility and has a scaled-up RVSS with more sites in governorates. There is a greater need for sensitivity enhancement of the RVSS. There is a need to ensure timely feedback from the WHO EMRO on the results of samples. Regular refresher training and feedback for health staff at the sentinel sites are recommended.

\section{Acknowledgments}

The authors would like to acknowledge support from the Training Programs in Epidemiology and Public Health Interventions Network (TEPHINET) and the Global Health Development, Eastern Mediterranean Public Health Network (GHD EMPHNET).

\section{Conflicts of Interest}

None declared.

\section{References}

1. Walker CLF, Rudan I, Liu L, Nair H, Theodoratou E, Bhutta ZA, et al. Global burden of childhood pneumonia and diarrhoea. Lancet 2013 Apr 20;381(9875):1405-1416 [FREE Full text] [doi: 10.1016/S0140-6736(13)60222-6] [Medline: 23582727]

2. World Health Organization, EMRO. Rotavirus gastroenteritis: Disease burden. URL: http://www.emro.who.int/health-topics/ rotavirus-gastroenteritis/disease-burden.html [accessed 2018-10-10]

3. World Health Organization. Rotavirus vaccines: WHO position paper: February 2013. Wkly Epidemiol Rec. 2013. URL: https://www.who.int/wer/2013/wer8805.pdf [accessed 2021-05-26]

4. Tate JE, Burton AH, Boschi-Pinto C, Parashar UD, World Health Organization-Coordinated Global Rotavirus Surveillance Network. World Health Organization? Coordinated Global Rotavirus Surveillance Network. Clin Infect Dis 2016 May 01;62 Suppl 2:S96-S105 [FREE Full text] [doi: 10.1093/cid/civ1013] [Medline: 27059362]

5. Malek M, Teleb N, Abu-Elyazeed R, Riddle M, Sherif M, Steele A, et al. The epidemiology of rotavirus diarrhea in countries in the Eastern Mediterranean Region. J Infect Dis 2010 Sep 01;202(Suppl):S12-S22 [FREE Full text] [doi: 10.1086/653579]

6. Ministry of Public Health and Population. Bacterial Meningitis and Rotavirus Program. Annual Report. In: Network Surveillance of Bacterial Meningitis and Rotavirus. Sana'a, Yemen: Ministry of Public Health and Population; 2017.

7. Amood Al-Kamarany M, Al-Areqi L, Mujally A, Alkarshy F, Nasser A, Jumaan AO. Diarrheal diseases hospitalization in Yemen before and after rotavirus vaccination. Scientifica (Cairo) 2016;2016:8485417 [FREE Full text] [doi: 10.1155/2016/8485417] [Medline: 27437161] 
8. German R, Lee L, Horan J, Milstein R, Pertowski C, Waller MN, Guidelines Working Group Centers for Disease Control Prevention (CDC). Updated guidelines for evaluating public health surveillance systems: Recommendations from the Guidelines Working Group. MMWR Recomm Rep 2001 Jul 27;50(RR-13):1-35; quiz CE1. [Medline: 18634202]

9. Roberts-Witteveen AR, Patel MS, Roche PW. An evaluation of the Australian Rotavirus Surveillance Program. Commun Dis Intell Q Rep 2008 Sep;32(3):326-332 [FREE Full text] [Medline: 19062768]

10. Muthama MR, Abade A, Amwayi S, Oundo J, Omolo J. Evaluation of Rotavirus Surveillance System - Kenya, 2010. TEPHINET. 2011. URL: https://www.tephinet.org/evaluation-of-rotavirus-surveillance-system-\%E2\%80\%94kenya-2010 [accessed 2021-05-02]

\author{
Abbreviations \\ CDC: Centers for Disease Control and Prevention \\ EIA: enzyme immunoassay \\ EMR: Eastern Mediterranean Region \\ MoPH\&P: Ministry of Public Health and Population \\ NCPHL: National Central Public Health Laboratory \\ PCR: polymerase chain reaction \\ PPV: positive predictive value \\ RV: Rotavirus \\ RVSS: Rotavirus Surveillance System \\ WHO: World Health Organization \\ WHO EMRO: WHO Regional Office for the Eastern Mediterranean
}

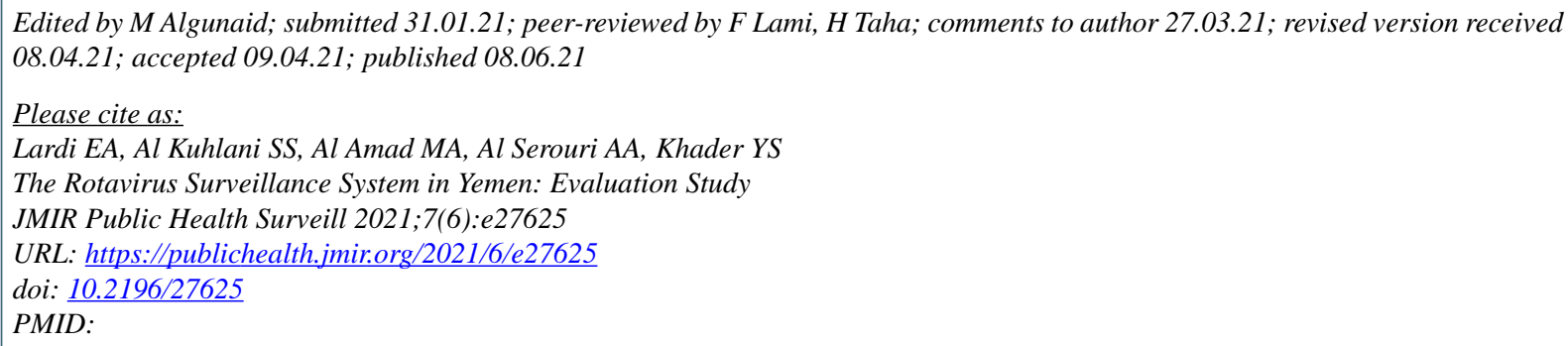

CEman Abdullah Lardi, Sharaf Sharaf Al Kuhlani, Mohammed Abdullah Al Amad, Abdulwahed Abduljabar Al Serouri, Yousef Saleh Khader. Originally published in JMIR Public Health and Surveillance (https://publichealth.jmir.org), 08.06.2021. This is an open-access article distributed under the terms of the Creative Commons Attribution License (https://creativecommons.org/licenses/by/4.0/), which permits unrestricted use, distribution, and reproduction in any medium, provided the original work, first published in JMIR Public Health and Surveillance, is properly cited. The complete bibliographic information, a link to the original publication on https://publichealth.jmir.org, as well as this copyright and license information must be included. 